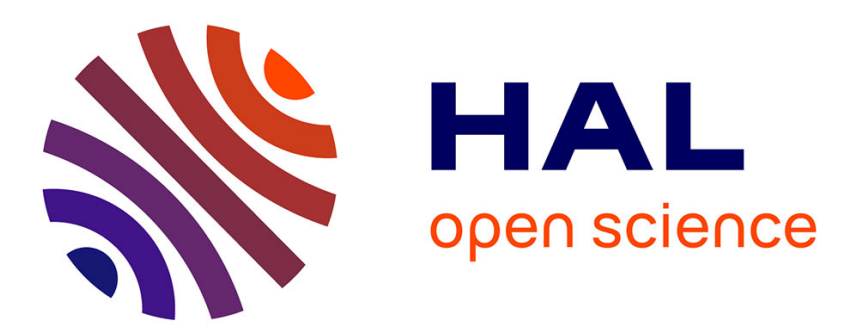

\title{
Nearly Perfect Fano Transmission Resonances through Nanoslits Drilled in a Metallic Membrane
}

Stéphane Collin, Grégory Vincent, Riad Haïdar, Nathalie Bardou, Sylvain Rommeluère, Jean-Luc Pelouard

\section{- To cite this version:}

Stéphane Collin, Grégory Vincent, Riad Haïdar, Nathalie Bardou, Sylvain Rommeluère, et al.. Nearly Perfect Fano Transmission Resonances through Nanoslits Drilled in a Metallic Membrane. Physical Review Letters, 2010, 104 (2), 10.1103/physrevlett.104.027401 . hal-03398798

\section{HAL Id: hal-03398798 https://hal.science/hal-03398798}

Submitted on 23 Oct 2021

HAL is a multi-disciplinary open access archive for the deposit and dissemination of scientific research documents, whether they are published or not. The documents may come from teaching and research institutions in France or abroad, or from public or private research centers.
L'archive ouverte pluridisciplinaire HAL, est destinée au dépôt et à la diffusion de documents scientifiques de niveau recherche, publiés ou non, émanant des établissements d'enseignement et de recherche français ou étrangers, des laboratoires publics ou privés. 


\title{
Nearly Perfect Fano Transmission Resonances through Nanoslits Drilled in a Metallic Membrane
}

\author{
Stéphane Collin, ${ }^{1, *}$ Grégory Vincent, ${ }^{1,2}$ Riad Haïdar, ${ }^{2}$ Nathalie Bardou, ${ }^{1}$ Sylvain Rommeluère, ${ }^{2}$ and Jean-Luc Pelouard ${ }^{1}$ \\ ${ }^{1}$ Laboratoire de Photonique et de Nanostructures (LPN-CNRS), Route de Nozay, 91460 Marcoussis, France \\ ${ }^{2}$ Office National d'Etudes et de Recherches Aérospatiales (ONERA), Chemin de la Hunière, 91761 Palaiseau, France
}

(Received 27 July 2009; revised manuscript received 8 December 2009; published 15 January 2010)

\begin{abstract}
We report nearly perfect optical transmission (87\%) through freestanding metallic gratings with narrow slits, as the experimental demonstration of the theoretical prediction by Porto et al. [Phys. Rev. Lett. 83, 2845 (1999)]. In addition, we show that the Fano line shape of transmission spectra reveals the interplay between localized and propagating surface plasmon resonances, and allows us to determine the nonradiative losses. It provides the limits for the transmission efficiency and resonance quality factor. As an illustration, a mosaic of various bandpass filters has been achieved in a single membrane.
\end{abstract}

Light-matter interactions in a metal layer patterned at the subwavelength scale give rise to a wide variety of optical resonances. It can lead to the excitation of either propagating surface plasmon polaritons (SPP) in metal films with hole arrays [1], or localized plasmon resonances in metamaterials composed of nanoscale resonators [2]. These phenomena allow us to design artificial materials having various refractive indices and spectral dispersion properties, and hold out the promise of the realization of planar optical elements like lenses [3] and filters [4,5]. However, their efficiency is hindered by the use of a substrate.

In contrast, perfect transmission and reflection is a general property of Fano resonances occurring in a symmetric slab made of lossless materials structured at the subwavelength scale [6]. However, the inclusion of metal components usually has a negative impact on both the transmission efficiency and the resonance width. Taking into account these losses, theoretical works have predicted nearly perfect optical transmission with resonance quality factors ranging over several orders of magnitude for freestanding metallic gratings with very narrow slits [7-10].

In this Letter, we report the demonstration of nearly perfect resonant optical transmission through freestanding metallic gratings made of core-shell membranes drilled with narrow slits. Measured transmission peaks are perfectly described by the predicted Fano line shape for the whole angular range $\left(0-40^{\circ}\right)$. Furthermore, we show that transmission spectra allow an experimental determination of the nonradiative losses, and provide the limits for the transmission efficiency and resonance quality factor. Such a structure has been used to realize a mosaic of various bandpass filters in a single membrane.

Freestanding transmission gratings made of thin metallic wires have already been fabricated and studied and have been used to act as nonresonant wire grid polarizers for more than one century [11-14]. However, narrow resonances with nearly perfect optical transmission have not been observed, since closely spaced wires with narrow $(\lambda / 4-\lambda / 10)$ and deep rectangular slits are required. Transmission efficiency and quality factors measured with metallic gratings deposited on dielectric substrate are also orders of magnitude lower than those predicted for symmetric structures $[15,16]$. Here, the fabrication of large-area freestanding metallic gratings with narrow slits has been achieved with a core-shell structure (see Fig. 1). A freestanding $\mathrm{Si}_{3} \mathrm{~N}_{4}$ membrane is fabricated on a $\mathrm{Si}$ substrate and drilled by dry etching [17]. Then, it is covered by a gold coating much thicker than the optical skin depth. The gold deposition is made in several steps at different incident angles, allowing a fine control of the geometry of the final structure. The mechanical properties of the whole structure are provided by the $\mathrm{Si}_{3} \mathrm{~N}_{4}$ core, leading to straight bars, whereas the optical properties are driven by the opaque gold shell: it behaves like a pure gold grating. Figure 2(a) shows a detail of the $3 \mathrm{~mm}^{2}$ freestanding metallic grating of thickness $t=2.6 \mu \mathrm{m}$, slit width $w=$ $2.7 \mu \mathrm{m}$, and period $d=9.65 \mu \mathrm{m}$.

The transmission diagram $T\left(\omega, k_{x}\right)$ is plotted in Fig. 2(b) where $\omega=2 \pi c / \lambda$ is the frequency, $\lambda$ is the wavelength, and $k_{x}=(\omega / c) \sin \theta$ is the incident wave vector $x$ component. The absolute transmission intensity is plotted in a linear grey scale. It has been determined experimentally by

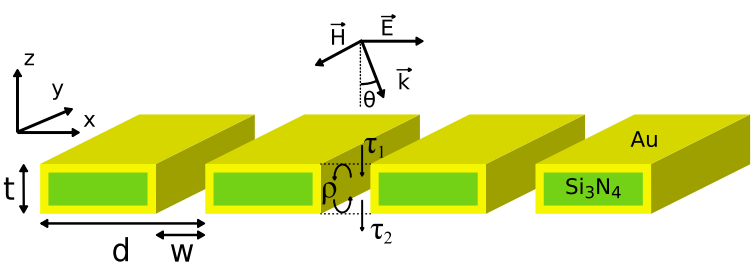

FIG. 1 (color online). Core-shell structure of freestanding metallic membranes with narrow slits. The mechanical properties of the structure are provided by the $\mathrm{Si}_{3} \mathrm{~N}_{4}$ core, whereas its optical properties are driven by the opaque gold shell. The schematic illustrates the geometrical parameters $(d, w, t)$, the incident transverse polarized plane wave (magnetic field along the wire axis), and the transmission $\left(\tau_{i}\right)$ and reflection $(\rho)$ coefficients. 


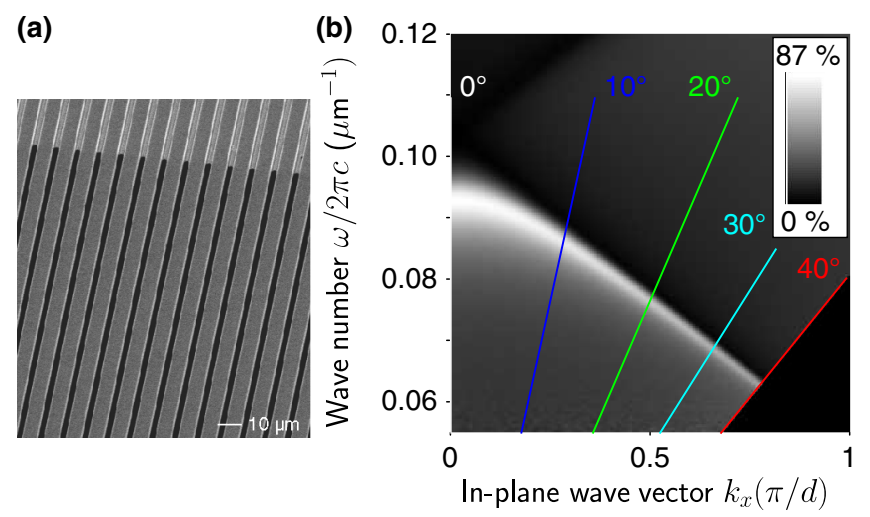

FIG. 2 (color online). Nearly perfect optical transmission through metallic membrane with subwavelength slits. (a) Scanning electron microscopy (SEM) image of the freestanding metallic membrane of thickness $t=2.6 \mu \mathrm{m}$, slit width $w=2.7 \mu \mathrm{m}$, and period $d=9.65 \mu \mathrm{m}$. (b) Transmission diagram measurement plotted on a linear gray scale.

angle-resolved transmission measurements performed with a Fourier-transform infrared spectrometer (Bruker Equinox $55 / S$ ) with liquid $\mathrm{N}_{2}$-cooled $\mathrm{InSb}$ and $\mathrm{HgCdTe}$ detectors. The spectral resolution is set to $5 \mathrm{~cm}^{-1}$. A homemade achromatic optical system allows $\Delta \theta=$ $\pm 0.5^{\circ}$ angular resolution [18]. Zero-order transmission spectra have been measured under an incidence angle ranging from $\theta=0^{\circ}$ to $\theta=40^{\circ}$ in $0.5^{\circ}$ increments, in TM polarization (i.e., magnetic field parallel to the slits).

It is remarkable that the absolute transmission intensity reaches $87 \%$ at normal incidence. Perfect transmission is predicted for ideal nonabsorbing metallic grating with rectangular slits, but both the transmission maximum and the quality factor are limited by metal absorption and fabrication imperfections. Hence, it is very difficult to predict theoretically the limitations of real structures. Figure 3(a) shows a comparison between measured transmission spectra (solid color curves) and exact numerical results (grey curves) for the five different incidence angles shown in Fig. 2(b). Numerical calculations were performed using the exact modal expansion and $S$-matrix formalism. The permittivity of gold was modelized by a Drude model: $\epsilon_{m}=1-\omega_{p}^{2} /\left(\omega^{2}+i \omega \gamma\right)$ with $\omega_{p}=1.15 \times 10^{16} \mathrm{~s}^{-1}$ and $\gamma=0.9 \times 10^{14} \mathrm{~s}^{-1}$ [9]. Measurements and calculations are in qualitative agreement. However, as discussed further, numerical results show slightly higher transmission efficiencies and smaller resonance widths.

The white band in Fig. 2(b) reveals a high-transmission resonance located below the first Rayleigh anomaly (defined as the low-frequency limit for the propagation of firstorder diffracted wave). The resonance exhibits low dispersion in the center of the Brillouin zone. For increasing incidence angle, the transmission resonance becomes much thinner and undergoes dispersion having clearly a surface resonance character. Two different mechanisms are involved. On the one hand, the direct coupling of incident light with vertical waveguide resonances in the slits exhib-

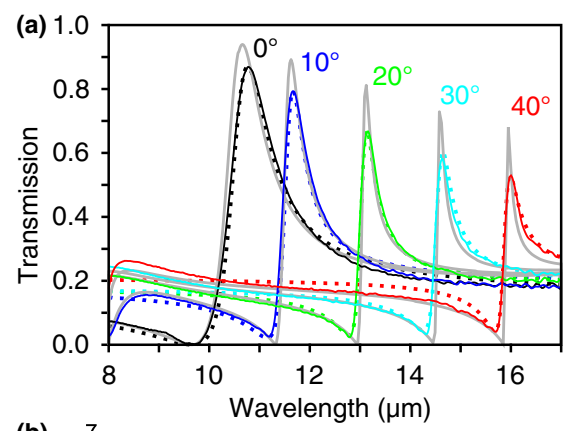

(b)

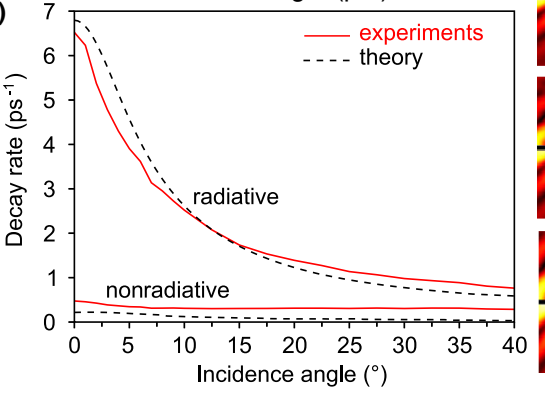

(c)

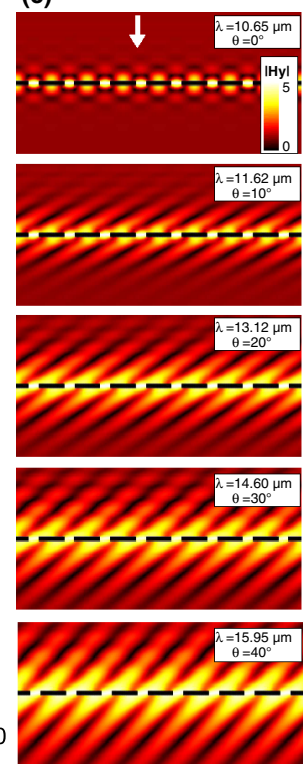

FIG. 3 (color online). Fano resonances. Radiative and nonradiative losses. (a) Comparison of transmission spectra for different incident angles: experiments (color solid curves), calculations (gray solid curves, higher maxima) and Fano model (color dashed curves). (b) Radiative and nonradiative decay rates determined from experimental data by a Fano fit (red solid curves), and from mode calculations (dashed curves). (c) Maps of the magnetic field amplitude calculated for the five resonances shown in (a). The amplitude is normalized to the incident field (linear color scale). White arrow: impinging light.

its flat dispersion curve and low quality factor. On the other hand, the excitation of horizontal surface plasmon waves on the upper and lower surfaces of the membrane contributes to strong dispersion and much higher quality factor. It is worth noticing that both mechanisms can be involved in the different parts of a single dispersion curve.

The Fano line shape of transmission spectra is related to the nature of the resonance mechanism. Since slits are much smaller than the wavelength, they support only one propagating waveguide mode (wave vector $\kappa$ ). Hence, the zero-order transmission intensity can be described by a simple one-mode Fabry-Perot model $[10,19,20]$ :

$$
T\left(\omega, k_{x}\right)=\left|\frac{\tau_{1} \tau_{2}}{1-\rho^{2} e^{2 i \kappa t}}\right|^{2}
$$

where $\tau_{i}$ and $\rho$ are the transmission and reflection coefficients at the interfaces (see Fig. 1). For a given $k_{x}$, the resonance condition can be written $1 / T\left(\omega_{p}\right)=0$ where $\omega_{p}=\omega_{p 0}+i \gamma$ is the complex frequency pole and $\gamma$ is the decay rate. The resonance quality factor is defined by $Q=$ $\omega_{p 0} /(2 \gamma)$. According to the Fabry-Perot model, the resonance condition can be fulfilled for any reflection coefficient $\rho$ and sufficiently thick gratings, leading to a Lorentzian-shape transmission intensity: $T(\omega)=\beta / \mid \omega-$ $\left.\omega_{p}\right|^{2}$. If no surface resonance occurs at the upper and lower 
interfaces, the reflection coefficient $\rho$ is low and slightly dispersive, resulting in nearly flat dispersion curves $\omega_{p}\left(k_{x}\right)$. In case of surface resonance, the transmission and reflection coefficients have a pole close to the Rayleigh anomaly, leading to a dispersive resonance. Because of this pole, $|\rho|$ increases, which induces much higher quality factors. In addition, the destructive interference between incident light and surface waves induces a complex zero $\omega_{0}$ of the transmission coefficients $\tau_{1}$ and $\tau_{2}$, in close proximity of their pole [20,21]. As a result, the transmission intensity can be approximated by a Fano model: $T(\omega)=\beta\left|\left(\omega-\omega_{0}\right) /\left(\omega-\omega_{p}\right)\right|^{2}$.

In summary, the pole $\omega_{p}$ originates from a Fabry-Perottype resonance of the waveguide mode into the slits, whatever surface resonances are involved. In our case, horizontal surface resonances play an important role. First, they induce dispersion and increase of the quality factor due to more reflective interfaces at the upper and lower ends of the slits. Second, they are responsible for a zero transmission close to the resonance. Indeed, the excellent agreement between the Fano model and experimental data shown in Fig. 3(a) confirms the role of surface phenomena in the transmission mechanism. It results in light localization both in the slits and on the (upper and lower) horizontal grating surfaces. As shown in Fig. 3(c), the weight of surface waves increases with incident angle, with the increase of both the magnetic field amplitude at the upper and lower surfaces, and the extension above and below the grating. The tricky interplay between localized and propagating SPP resonances allows us to tune the quality factor over a wide range by changing the slit width and period. We emphasize that, in the present case, Fano line shape results from two different resonant mechanisms. In contrast, in metal films with hole arrays the Fano line shape is usually attributed to the interference of resonant and nonresonant processes [22]. Also, in the case of nonsymmetric metallic grating, surface plasmon resonances can not be excited simultaneously on the upper and lower surfaces of the grating. It results in much weaker interactions between localized and propagating SPP resonances, leading to much lower transmission efficiency and quality factors $[15,16]$.

The total decay rate is the sum of the radiative decay rate (zero-order transmission and reflection) and the nonradiative decay rate (absorption and scattering): $\gamma=\gamma_{r}+\gamma_{n r}$. The fit of the Fano model to the experimental data, done by adjusting the parameters $\beta, \omega_{0}$, and $\omega_{p}$, provides a precise determination of $\gamma$. Besides, it was shown theoretically that the transmission maximum can be expressed as $T_{\max }=\left|\gamma_{r} /\left(\gamma_{r}+\gamma_{n r}\right)\right|^{2}$ [23]. As a result, the radiative and nonradiative decay rates can be determined from experimental data for the whole dispersion curve, as shown in Fig. 3(b). The radiative decay rate is in quantitative agreement with numerical calculations. It is found to be more than 1 order of magnitude larger than the nonradiative decay rate at normal incidence. This is the origin of the nearly perfect optical transmission measured with this sample. As we should expect, the nonradiative losses are underestimated by the model (we find a factor of 2 at normal incidence). This difference is mainly attributed to metal absorption and light scattering due to metal roughness and inhomogeneities. The decrease of the radiative decay rate along the dispersion curve is related to the increasing weight of surface resonance mechanisms [23], leading to experimental quality factor ranging from 13 for $\theta=0^{\circ}$ to 56 for $\theta=40^{\circ}$.

These results open a way to the design of arrays of highefficiency bandpass spectral filters in a single layer. As already stated above, the main transmission mechanism is based on a tunable Fabry-Perot resonance in the slits. The transmission peak position $\lambda_{p}$ is adjusted by the choice of $d\left(d \simeq \lambda_{p}\right)$, impacting the phase of the reflection coefficient $\rho$. Since both (horizontal and vertical) resonances shift with $d$, it is necessary to adjust the resonance width $\Delta \lambda=\lambda_{p} / Q$ by tuning the slit width $w / d \simeq 0.15$.

A mosaic of filters dedicated to multispectral imaging has been fabricated in a $20 \mathrm{~mm}^{2}$ core-shell membrane of total thickness $t=950 \mathrm{~nm}$ [see Figs. 4(a) and 4(b)]. It is divided into ten $1.5 \times 1.7 \mathrm{~mm}^{2}$ gratings designed to act as ten bandpass filters regularly spaced in the 3-5 $\mu \mathrm{m}$ wavelength range, with periods in the $2.45-4.7 \mu \mathrm{m}$ range and aperture fill factors in the $0.12-0.16$ range. Crossing struts (width $500 \mathrm{~nm}$ ) with a $40 \mu \mathrm{m}$ period improve the structure stiffness with negligible optical impact, so that it still behaves as a one-dimensional grating. The membrane is
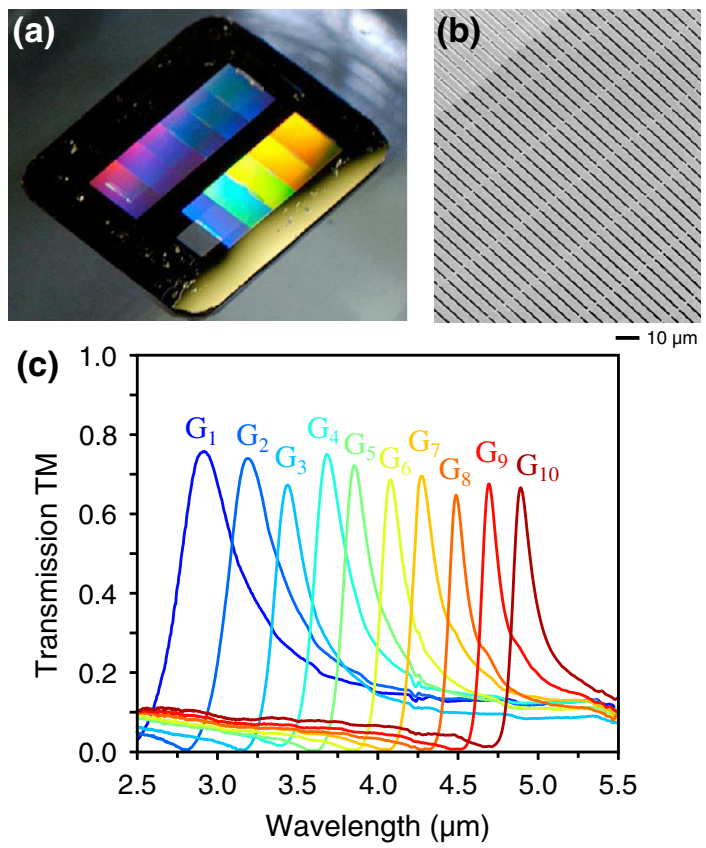

FIG. 4 (color online). Mosaic of bandpass filters in a single $950 \mathrm{~nm}$-thick membrane. (a) Optical and (b) SEM images of the $20 \mathrm{~mm}^{2}$ membrane. (c) Measured transmission spectra of the ten bandpass filters at normal incidence. The geometrical parameters of the filters are given in Table I. 
TABLE I. Geometrical parameters and experimental results $\left(\lambda_{\max }, Q\right)$ of the 10 membrane filters. Each membrane extends over $1.5 \mathrm{~mm} \times 1.7 \mathrm{~mm}$. The thickness is $950 \mathrm{~nm}$.

\begin{tabular}{ccccccr}
\hline \hline Grating & $d_{i}(\mu \mathrm{m})$ & $w_{i}(\mu \mathrm{m})$ & $\alpha_{i}=d_{i} / w_{i}$ & $T_{\max }$ & $\lambda_{\max }(\mu \mathrm{m})$ & $Q$ \\
\hline$G_{1}$ & 2.45 & 0.32 & 0.131 & 0.76 & 2.92 & 8.6 \\
$G_{2}$ & 2.80 & 0.36 & 0.129 & 0.74 & 3.18 & 11.7 \\
$G_{3}$ & 3.15 & 0.39 & 0.124 & 0.67 & 3.43 & 16.7 \\
$G_{4}$ & 3.40 & 0.47 & 0.138 & 0.75 & 3.68 & 18.4 \\
$G_{5}$ & 3.60 & 0.48 & 0.133 & 0.72 & 3.85 & 23.0 \\
$G_{6}$ & 3.85 & 0.50 & 0.13 & 0.68 & 4.08 & 26.6 \\
$G_{7}$ & 4.05 & 0.61 & 0.151 & 0.70 & 4.27 & 27.7 \\
$G_{8}$ & 4.30 & 0.55 & 0.128 & 0.65 & 4.49 & 37.6 \\
$G_{9}$ & 4.50 & 0.69 & 0.153 & 0.68 & 4.6 & 37.9 \\
$G_{10}$ & 4.70 & 0.77 & 0.164 & 0.67 & 4.8 & 37.7 \\
\hline \hline
\end{tabular}

removed in an additional $1.5 \times 1.7 \mathrm{~mm}^{2}$ window to serve as a reference. Measured transmission spectra at normal incidence are shown in Fig. 4(c). We obtain regularly spaced filters over the $3-5 \mu \mathrm{m}$ spectral range $(\delta \lambda=$ $220 \mathrm{~nm} \pm 50 \mathrm{~nm})$ and high-transmission maxima $(70 \% \pm$ $5 \%)$. The quality factors have been designed in order to obtain resonance widths close to the spectral spacing $\delta \lambda$. It has to be emphasized that, in practice, such filter arrays are not feasible with conventional multilayer filters due to their huge number of layers and their large thicknesses required in the midinfrared domain.

In summary, we have demonstrated nearly perfect optical transmission through freestanding metallic gratings made of core-shell membranes with narrow slits. The Fano line shape of transmission spectra originates from the interplay between cavity resonances in the slits and surface plasmon resonances on the horizontal grating surfaces. It allows an experimental determination of both radiative and nonradiative losses. A mosaic of spectral filters has been fabricated in a single metallic membrane patterned at the subwavelength scale. It exhibits $70 \%$ maximum transmission efficiency in $15 \%$ aperture area, which represents a fivefold enhancement compared to the geometrical transmission. This mosaic of bandpass filters can be used for spectral imaging in the infrared domain. This work could also be extended to freestanding dielectric gratings acting as band-stop filters, with a perfect reflection predicted at the resonant wavelength [24,25]. In the near future, such nanostructured membranes shall pave the way to conception of a new class of high-efficiency and compact optical elements arrays (i.e., polarizer, filter, or lens arrays) that can be integrated in the vicinity of focal plane arrays.

We acknowledge helpful discussions with F. Pardo, N. Guérineau, J. Primot, P. Lalanne, and C. Sauvan.

*Stephane.Collin@1pn.cnrs.fr

[1] W. L. Barnes, A. Dereux, and T.W. Ebbesen, Nature (London) 424, 824 (2003).
[2] V. M. Shalaev, Nat. Photon. 1, 41 (2007).

[3] L. Verslegers, P. B. Catrysse, Z. Yu, J. White, S. Edward, S. Barnard, M. L. Brongersma, and S. Fan, Nano Lett. 9, 235 (2009).

[4] T. W. Ebbesen, H. J. Lezec, H. F. Ghaemi, T. Thio, and P. A. Wolff, Nature (London) 391, 667 (1998).

[5] E. Laux, C. Genet, T. Skauli, and T. W. Ebbesen, Nat. Photon. 2, 161 (2008).

[6] E. Popov, L. Mashev, and D. Maystre, Opt. Acta 33, 607 (1986).

[7] J. A. Porto, F. J. García-Vidal, and J. B. Pendry, Phys. Rev. Lett. 83, 2845 (1999).

[8] S. Collin, F. Pardo, R. Teissier, and J.-L. Pelouard, Phys. Rev. B 63, 033107 (2001).

[9] S. Collin, F. Pardo, R. Teissier, and J.-L. Pelouard, J. Opt. A 4, S154 (2002).

[10] P. Lalanne, J. P. Hugonin, S. Astilean, M. Palamaru, and K. D. Möller, J. Opt. A 2, 48 (2000).

[11] H. Hertz, Electric Waves (Macmillan and Company, Ltd., London, 1893).

[12] G. R. Bird and M. Jr. Parrish, J. Opt. Soc. Am. 50, 886 (1960).

[13] H. Lochbihler, Phys. Rev. B 50, 4795 (1994).

[14] H. Lochbihler, E. Polnau, and P. Predehl, Appl. Opt. 34, 5725 (1995).

[15] Z. Sun, Y.S. Jung, and H. K. Kim, Appl. Phys. Lett. 83, 3021 (2003); H.E. Went, A.P. Hibbins, J. R. Sambles, C. R. Lawrence, and A. P. Crick, Appl. Phys. Lett. 77, 2789 (2000); A. Barbara, P. Quémerais, E. Bustarret, and T. Lopez-Rios, Phys. Rev. B 66, 161403(R) (2002); K. G. Lee and Q-Han Park, Phys. Rev. Lett. 95, 103902 (2005).

[16] C. Ropers, D. J. Park, G. Stibenz, G. Steinmeyer, J. Kim, D. S. Kim, and C. Lienau, Phys. Rev. Lett. 94, 113901 (2005); Y. Pang, C. Genet and T. W. Ebbesen, Opt. Commun. 280, 10 (2007); D. Pacifici, H. J. Lezec, Harry A. Atwater, and J. Weiner, Phys. Rev. B 77, 115411 (2008); C. Billaudeau, S. Collin, F. Pardo, N. Bardou, and J. L. Pelouard, Opt. Express 17, 3490 (2009).

[17] G. Vincent, S. Collin, N. Bardou, J.L. Pelouard, and R. Haïdar, J. Vac. Sci. Technol. B 26, 1852 (2008).

[18] C. Billaudeau, S. Collin, C. Sauvan, N. Bardou, F. Pardo, and J. L. Pelouard, Opt. Lett. 33, 165 (2008).

[19] F. J. García-Vidal and L. Martín-Moreno, Phys. Rev. B 66, 155412 (2002).

[20] P. Lalanne, C. Sauvan, J. P. Hugonin, J. C. Rodier, and P. Chavel, Phys. Rev. B 68, 125404 (2003).

[21] D. Maystre, Electromagnetic Surface Modes (Wiley, New York, 1982), Chap. 17.

[22] C. Genet, M. van Exter, and J. Woerdman, Opt. Commun. 225, 331 (2003); M. Sarrazin, J. P. Vigneron, and J. M. Vigoureux, Phys. Rev. B 67, 085415 (2003); W. Fan, S. Zhang, B. Minhas, K. J. Malloy, and S. R. J. Brueck, Phys. Rev. Lett. 94, 033902 (2005); J. Bravo-Abad, L. MartínMoreno, F. J. García-Vidal, E. Hendry, and J. Gómez Rivas, Phys. Rev. B 76, 241102(R) (2007).

[23] F. Marquier, J. J. Greffet, S. Collin, F. Pardo, and J. L. Pelouard, Opt. Express 13, 70 (2005).

[24] R. Gómez-Medina, M. Laroche, and J. J. Sáenz, Opt. Express 14, 3730 (2006).

[25] M. Laroche, S. Albaladejo, R. Gómez-Medina, and J. J. Sáenz, Phys. Rev. B 74, 245422 (2006). 